\title{
Adjunctive air-polishing with erythritol in nonsurgical periodontal therapy: a randomized clinical trial
}

\author{
Holger F. R. Jentsch ${ }^{*^{*}}$, Christian Flechsig ${ }^{2}$, Benjamin Kette ${ }^{2}$ and Sigrun Eick ${ }^{3}$
}

\begin{abstract}
Background: This study was aimed to investigate if the adjunctive use of erythritol air-polishing powder applied with the nozzle-system during subgingival instrumentation (SI) has an effect on the outcome of non-surgical periodontal treatment in patients with moderate to severe periodontitis.

Methods: Fourty-two individuals with periodontitis received nonsurgical periodontal therapy by SI without (controls, $n=21$ ) and with adjunctive air-polishing using nozzle + erythritol powder (test, $n=21$ ). They were analyzed for the clinical variables BOP (primary outcome at six months), probing depth (PD), attachment level, four selected microorganisms and two biomarkers at baseline, before SI as well as three and six months after SI. Statistical analysis included nonparametric tests for intra- and intergroup comparisons.

Results: In both groups, the clinical variables PD, attachment level and BOP significantly improved three and six months after SI. The number of sites with PD $\geq 5 \mathrm{~mm}$ was significantly lower in the test group than in the control group after six months. At six months versus baseline, there were significant reductions of Tannerella forsythia and Treponema denticola counts as well as lower levels of MMP-8 in the test group.

Conclusions: Subgingival instrumentation with adjunctive erythritol air-polishing powder does not reduce BOP. But it may add beneficial effects like reducing the probing depth measured as number of residual periodontal pocket with $\mathrm{PD} \geq 5 \mathrm{~mm}$ when compared with subgingival instrumentation only.

Clinical relevance: The adjunctive use of erythritol air-polishing powder applied with the nozzle-system during SI may improve the clinical outcome of SI and may reduce the need for periodontal surgery.

Trial registration The study was retrospectively registered in the German register of clinical trials, DRKS00015239 on 6th August 2018, https://www.drks.de/drks_web/navigate.do?navigationld=trial.HTML\&TRIAL.
\end{abstract}

Keywords: Periodontitis, Subgingival instrumentation, Clinical variables, Subgingival microorganisms, Erythritol, Biomarker

*Correspondence: jenh@medizin.uni-leipzig.de

${ }^{1}$ Department of Cariology, Endodontology and Periodontology, Centre for Periodontology, University Hospital of Leipzig, Liebigstr. 12, Haus 1, 04103 Leipzig, Germany

Full list of author information is available at the end of the article

\section{Background}

Periodontitis is not a simple bacterial infection but a complex disease where the subgingival microbiota, the immune conditions of the host and factors of the environment interact [1]. Beside the dental plaque exist other modifying factors that contribute to develop periodontal disease [2,3]. Nevertheless the supra- and subgingival bacterial biofilm is still the substrate that is 
accessable for the dental practitioner. The removal of the biofilm solves the dysbiosis with the overgrowth of more virulent microorganisms in the biofilm to restart eubiosis and to reduce inflammation [4].

Subgingival instrumentation (SI) to remove the biofilm and calculus is the cornerstone to perform a causative periodontal treatment [5]. Here, the removal of the biofilm with hand and/or sonic/ultrasonic instruments has been postulated as the gold standard for the treatment of periodontitis for many years $[6,7]$. Considerable improvements of bleeding on probing (BOP), probing pocket depth (PD) and clinical attachment level (AL) to avoid tooth loss due to periodontitis can be achieved [6]. Nevertheless several attempts-like additional use of lasers, photodynamic therapy and several antimicrobials-have been made to further improve the results of subgingival instrumentation.

New tools and substances to remove the biofilm from the tooth surface have been developed. Recently erythritol as an air-polishing substance has been introduced into the spectrum of treatment modalities. Subgingival air-polishing with glycine has been shown to be potentially more effective to remove the subgingival biofilm than hand and ultrasonic instruments [8]. Erythritol is a sugar alcohol (meso-1,2,3,4-Butantetrol). It is a water-soluble, chemically neutral artificial sweetener [9]. In relation to glycine, another small particle size air-polishing substance, erythritol has a smaller particle size [10]. Erythritol is suitable to remove effciently the subgingival biofilm from the root surface [10]. In addition, in vitro erythritol suppresses the formation of a dual-species biofilm of Porphyromonas gingivalis and Streptococcus gordonii via RNA and DNA depletion and metabolic changes [11]. Erythritol is also clinically appreciated due to patient's comfort and time efficiency [12].

Subgingival instrumentation in the initial therapy needs the removal of subgingival calculus using hand and/or sonic/ultrasonic instruments. However, there are only limited data if an adjunctive use of air-polishing with erythritol is beneficial for the clinical outcome during initial subgingival instrumentation [13, 14]. Following, the aim of the present randomized clinical trial was to verify, if the adunctive use of erythritol as an airpolishing mean for the removal of the subgingival biofilm during subgingival instrumentation gives superior results in comparison to conventional SI. The hypothesis of the study was that the adjunctive use of an airpolishing device using erythritol during SI results in a significant better outcome of the SI regarding clinical, microbiological and biomarker variables in comparison with the conventional SI six months after SI.

\section{Methods}

Study design

The study was approved by the Ethics Commission (\#AZ436/16-ek) of the Medical Faculty of the University of Leipzig. It is registered in the German register of clinical trials (DRKS00015239), and the full study protocol is deposited there. The clinical study was conducted as a randomized controlled trial with parallel design (two independent groups) in a private dental practice. The examiner as well as the laboratory personnel were blinded. Seventy-two patients were randomly selected, screened and asked to participate in the study. Fourtynine patients ( 30 male and 19 female) were willing and gave their written informed consent. The clinical trial was conducted in a private dental practice (Berlin, Germany). The principles outlined in the Declaration of Helsinki, as revised in 2008, were followed to obtain the informed consents and to conduct the clinical study. Patients with moderate to severe chronic periodontitis were included in the study [15] corresponding to stage II-III, grade B of the new classification scheme for periodontal and periimplant diseases and conditions [16].

The same experienced dentist (C. F.) performed all SIs in both study groups. All assessment of the clinical data as well as the collection of biofilm and gingival crevicular fluid (GCF) was performed by a second experienced dentist (B. K.) blinded to the study groups. The intraexaminer calibration for reliability testing resulted in $\kappa=0.91$ for repeated measurements of PD and AL in two quadrants of eight patients. A computer-generated randomization table was used for the recruitment and blinded the randomization of 49 participants either to the test $(n=24)$ or control groups $(n=25)$ with a 1:1 allocation ratio. An assistant of the dental practice performed the assignment to interventions and the documentation. To obtain the allocation concealment a sealed opaque envelope was used. The envelope contained the treatment number of the allocation table to the specific subject. The examiner did not perform the treatment procedures and was unaware of the treatment assignment.

\section{Participants of the study}

The inclusion criteria were age between 40 and 65 years, at least 16 natural teeth in function, periodontitis with probing depth $\leq 6 \mathrm{~mm}$, at least 16 teeth with need for SI, interproximal plaque index (API) [17] $\leq 35 \%$ at the baseline appointment after two appointments of professional prophylaxis with motivation and instruction within three weeks, no diseases with influence on the periodontal disease, no diabetes mellitus, arthritis or allergies on used substances or products. Patients were excluded if they were pregnant or breastfeeding, if they were 
smokers with more than seven cigarettes per day, if they had a treatment with antibiotics within the six months prior to the study, need for periodontal surgery or adjunctive antibiotic treatment to the SI and if they had any periodontal treatment during the last year before the study. Based on the results of Chondros et al. [18] for BOP with a difference of $11 \%$ at baseline a minimum of 20 volunteers per group would be necessary to detect a significant difference $(p \leq 0.05)$ with a test power of $80 \%$.

\section{Clinical procedures and sampling methods}

In the test and control groups the clinical variables were recorded at three appointments: at baseline before SI (t1) as well as three (t2) and six months (t3) after SI. The GCF samples and the samples of subgingival biofilm were collected at the same time. The participants received fullmouth SI at sites with $\mathrm{PD} \geq 4 \mathrm{~mm}$ in two sessions carried out within $24 \mathrm{~h}$ using hand and sonic instruments $\mathrm{CH}-$ Friedy Manufacturing Co., Chicago, IL, USA and Dentsply Sirona, Bensheim, Germany) under local anaesthesia with articaine hydrochloride/epinephrine hydrochloride (Ultracain D-S, Sanofi-Aventis, Frankfurt/Main, Germany). The criterium was a bioacceptable root surface without clinically detectable nonmineralized and mineralized material on the root surface after SI. In addition in the test group, erythritol powder (Air-Flow ${ }^{\circledR}$ Plus Powder, EMS Nyon, Switzerland) was applied for five seconds per site using the Perioflow ${ }^{\circledR}$ handpiece with the Perioflow ${ }^{\circledR}$ nozzle with the Air-Flow ${ }^{\circledR}$ Master apparatus (all EMS Nyon, Switzerland) respecting the recommendations of the manufacturer. The nozzle was introduced into the periodontal pocket as described by Hägi et al. [12]. The powder was directed perpendicular to the root surface. Via inclination of the handpiece as well as via the flexibility of the plastic nozzle device the access of interproximal buccal and oral sites was possible and the bottom of the pocket (inclusion criteria $\leq 6 \mathrm{~mm}$ ) could be reached.

The use of CHX has been performed as described in the studies of Jentsch et al. [19, 20]. During the first seven days after SI all patients used a chlorhexidine digluconate mouthrinse (Chlorhexamed forte $0.2 \%$, GlaxoSmithKline Healthcare, Bühl, Germany) for one minute twice daily. Using chlorhexidine digluconate after SI is recommended by the EFP S3 level guideline [21]. After SI careful normal oral hygiene using toothbrush and interdental brushes was performed. An appointment of comprehensive supportive periodontal therapy with removal of biofilm and calculus, (applying the erythritol powder with the Perioflow handpiece in the test group) as well as re-motivation and re-instruction took place three months after SI in both groups. In Fig. 1 the timeline of the study is presented.

In a six-point measurement per tooth (mesiobuccal, buccal, distobuccal, mesiooral, oral and distooral) the clinical variables $\mathrm{PD}, \mathrm{CAL}$ and $\mathrm{BOP}$ of all teeth were recorded. For the assessment a manual periodontal probe (PCP-UNC 15, Hu-Friedy Manufacturing Co., Chicago, IL, U.S.A.) using a pressure of $0.25 \mathrm{~N}$ was used. The oral hygiene was recorded by using the API [17].

At each appointment, samples of the GCF and the subgingival biofilm were taken from the deepest site per quadrant at baseline. To sample GCF for the analysis of the biomarkers, sterile paper strips (Periopaper; Oraflow Inc., Smithtown, New York, U.S.A.) were placed at the entrance of the periodontal pocket for $30 \mathrm{~s}$. As described by Griffiths [22] this intracrevicular superficial method avoids the destruction of the subgingival biofilm in the periodontal pocket. After pooling the paper strips were placed into a tube with $100 \mu \mathrm{l}$ protease inhibitor solution (Sigma Aldrich Chemie GmbH, München, Germany).

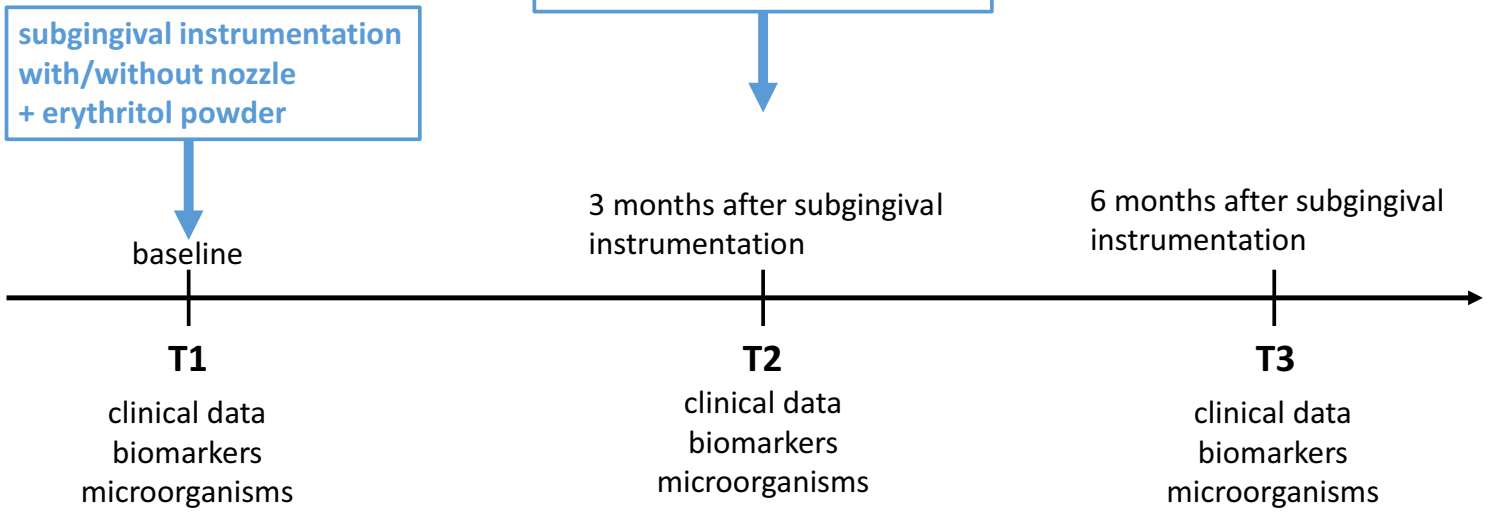

Fig. 1 Timeline of the study using adjunctive air-polishing with erythritol in nonsurgical periodontal therapy 
To collect the subgingival biofilm at the same sites as for GCF sampling endodontic paper points (ISO 60, Roeko $\mathrm{GmbH}$, Langenau, Germany) were inserted into the pocket until resistance was felt. The paper points were left in place for $30 \mathrm{~s}$. The GCF samples were stored at $-80^{\circ} \mathrm{C}$ and the biofilm samples at $-20^{\circ} \mathrm{C}$ until analysis.

\section{Laboratory analysis}

Before analysis, the GCF samples were eluted overnight into $650 \mu \mathrm{l}$ phosphate-buffered saline (+ proteinase inhibitors solution) at $4{ }^{\circ} \mathrm{C}$. From the eluates, the levels of interleukin (IL)- $1 \beta$ and matrix-metalloproteinase (MMP)-8 were determined by using commercially available enzyme-linked immunosorbent assay (ELISA) kits (R \& D Systems Europe Ltd., Abingdon, UK) according to the manufacturer's instructions. The detection levels were $2 \mathrm{pg} / \mathrm{site}$ for IL- $1 \beta$ and $100 \mathrm{pg} / \mathrm{site}$ for MMP-8.

For microbiological analysis, DNA was extracted and a multiplex-realtime qPCR for Aggregatibacter actinomycetemcomitans, Porphyromonas gingivalis, Tannerella forsythia and Treponema denticola was performed as described recently [19]. The results are given as bacterial counts $\log ^{10}$.

\section{Statistical analysis}

The primary outcome variable of the study was BOP six months after SI. Secondary outcome variables were changes of BOP after three months as well as PD, AL, of the number of sites with $\mathrm{PD} \geq 5 \mathrm{~mm}$, counts of the four selected periodontopathogenic bacteria, the levels of IL-1 $\beta$ and MMP- 8 three and six months after the SI.

The statistical analysis of all clinical and laboratory data was performed with the help of the software SPSS ${ }^{\circledR}$ Statistics 24.0 (IBM Corporation, New York, NY, U.S.A.). The Fisher's exact test compared qualitative data. Nonparametric tests (Friedman-test for comparing several time-points within a group, Wilcoxon test for paired samples and Mann Whitney U-test for inter-group comparisons, respectively) without testing for normal distribution were used for intra- and inter-group comparisons. The unit of analysis in all statistical tests was the individual participant. The level of significance was $\alpha \leq 0.05$.

\section{Results}

The study was performed from March 2017 until June 2018. In June 2018, the last patient left the study. The study flow adapted to Moher et al. [23] is presented in Fig. 2. Seventy-two patients were assessed for eligibility, 23 patients were excluded, seven patients were lost during the follow-up period. In Table 1 the demographic and baseline data of the 49 included patients are presented. There were no significant differences between both groups at baseline at the clinical variables. During the study no adverse effects of the different treatment procedures occurred and no additional medical treatment or drug intake were reported. After three months one patient was lost in the test group and three patients were lost in the control group. After six months two more patients were lost in the test group and one more patient was lost in the control group. The records of 42 participating patients with the clinical and laboratory data of all three appointments were available for statistical analysis. In Table 2 the changes of the clinical data during the study inclusive the statistical analysis are presented.

The means of $\mathrm{PD}, \mathrm{AL}$ and $\mathrm{BOP}$ were significantly improved in both groups three and six months after SI $(p<0.01, p<0.001)$ without any difference between the groups. Also the numbers of sites with $\mathrm{PD} \geq 5 \mathrm{~mm}$ decreased in both groups at three months and six months (each $p<0.001$ ). The number of sites with $\mathrm{PD} \geq 5 \mathrm{~mm}$ was significantly lower in the test group than in the control group after six months $(p=0.019)$. Only in the control group, the oral hygiene index improved at six month, but there was no significant difference between the groups at any time-point.

The levels of MMP-8 in the GCF decreased in the test group, they were significantly less at six months when compared with baseline, in the control group there was no change. The levels of IL-1 $\beta$ did not change, neither in the test nor in the control groups (Fig. 3). There was no statistical significant difference between the two groups at the studied biomarkers at any time.

Among the selected bacteria, $A$. actinomycetemcomitans was detected only rarely. $P$. gingivalis counts were decreased in the control group at three months versus baseline. The counts of $T$. forsythia and T. denticola were found to be reduced at six months versus baseline in the test group (Fig. 4). There was no statistical significant difference of the counts of the selected bacteria between the two groups at any time.

\section{Discussion}

In the present study analyzing the effect of additional airpolishing of the root surfaces with nozzle and erythritol powder during subgingival instrumentation the number of sites with $P D \geq 5 \mathrm{~mm}$ was significantly lower after additional air-polishing.

Reviewing the existent literature there are only limited data on the efficacy of using air-polishing with erythritol during periodontal therapy. Some studies focused on the effects of air-polishing with erythritol during the supportive periodontal therapy [10, 12, $24,25]$. Comparable results regarding $\mathrm{BOP}$ and $\mathrm{PD}$ as well as residual pockets $\geq 4 \mathrm{~mm}$ were reported for the nozzle use with erythritol and hand instruments, an advantage might be the low abrasiveness $[10,12]$. 


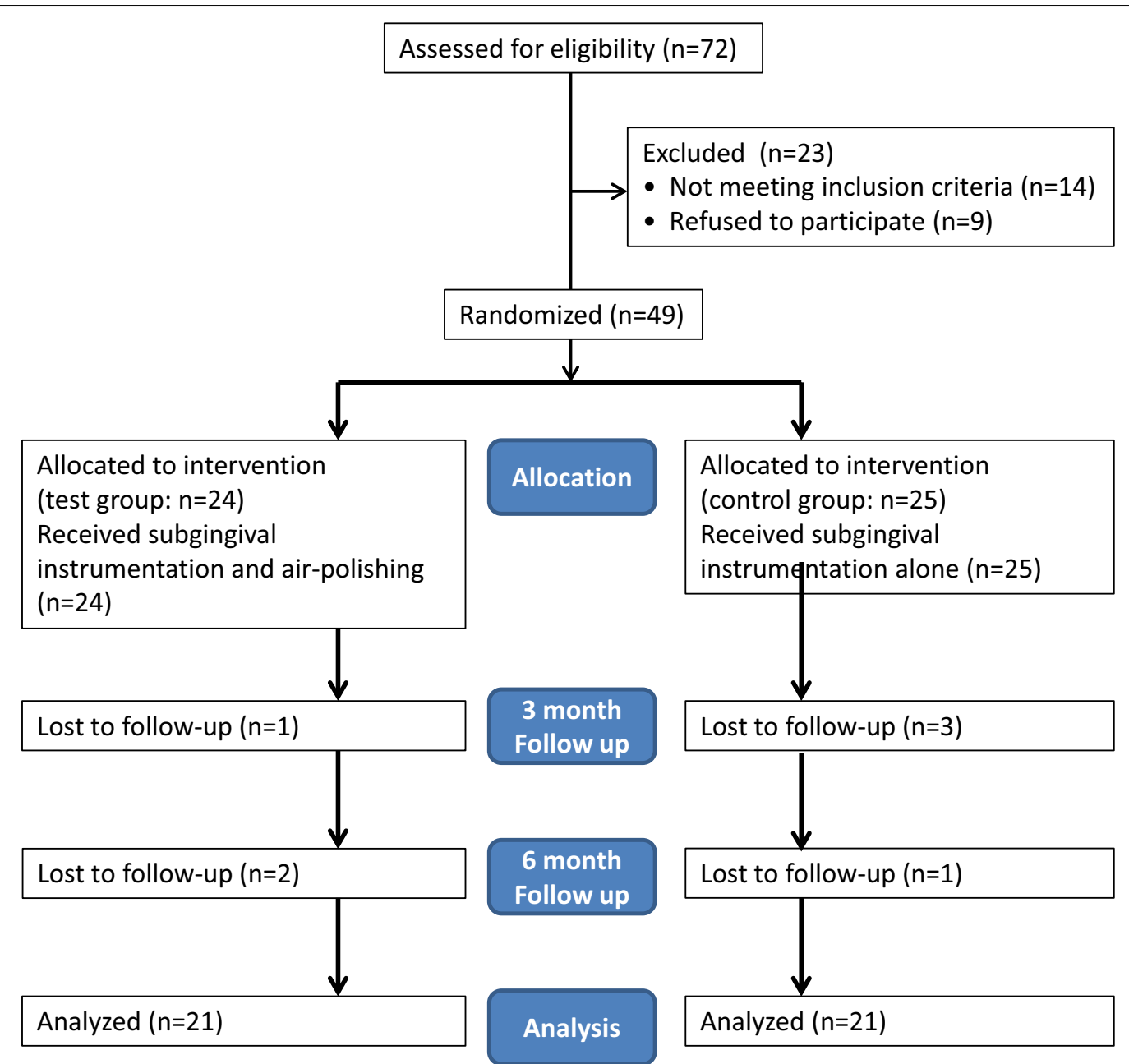

Fig. 2 Flowchart (adapted to Moher et al. 2001) of the study using adjunctive air-polishing with erythritol in nonsurgical periodontal therapy

Table 1 Demographic data in the test (subgingival instrumentation + subgingival erythritol air-polishing) and control (subgingival instrumentation alone) groups at baseline

\begin{tabular}{llll}
\hline Variable & Test group $(\mathbf{n = 2 1 )}$ & Control group $(\mathbf{n}=\mathbf{2 1})$ & U-test $(\mathbf{p})$ \\
\hline Age (years; mean \pm SD) & $50.23 \pm 8.26$ & $54.29 \pm 7.44$ & 0.144 \\
Male/female $(n)$ & $14 / 7$ & $11 / 10$ & $0.537^{\text {a }}$ \\
Smoker & 7 & 3 & $0.281^{\text {a }}$ \\
number of teeth (mean \pm SD) & $25.86 \pm 3.53$ & $26.00 \pm 3.08$ & 0.949 \\
PD (mm, mean \pm SD) & $3.14 \pm 0.39$ & $3.08 \pm 0.36$ & 0.697 \\
number of PD $\geq 5$ mm (mean \pm SD) & $29.88 \pm 15.27$ & $27.62 \pm 6.54$ & 0.667 \\
CAL (mm, mean $\pm S D)$ & $3.50 \pm 0.45$ & $3.52 \pm 0.44$ & 0.857 \\
BOP $(\%$, mean \pm SD) & $28.8 \pm 31.6$ & $27.9 \pm 16.5$ & 0.529 \\
API (\%, mean $\pm S D)$ & $16.9 \pm 9.7$ & $24.3 \pm 20.7$ & 0.412 \\
\hline
\end{tabular}

SD standard deviation, $n$ number

a Fisher's exact test, all other data Mann Whitney test 
Table 2 Clinical variables (mean $\pm S D$ ) of the test group (subgingival instrumentation + subgingival erythritol airpolishing) and control group (subgingival instrumentation alone) at baseline (T1) as well as three (T2) and six months (T3) after subgingival instrumentation incl. statistics

\begin{tabular}{|c|c|c|c|}
\hline $\begin{array}{l}N_{\text {test group }}=21 \\
N_{\text {control group }}=21\end{array}$ & T1, baseline & $\begin{array}{l}\text { T2, } \\
\text { after } 3 \text { months }\end{array}$ & $\begin{array}{l}\text { T3, } \\
\text { after } 6 \text { months }\end{array}$ \\
\hline \multicolumn{4}{|l|}{ Mean PD (mm) } \\
\hline Test group & $3.14 \pm 0.39$ & $2.44 \pm 0.42^{* * *}$ & $2.23 \pm 0.25^{* * *}$ \\
\hline Control group & $3.08 \pm 0.36$ & $2.46 \pm 0.35^{* * *}$ & $2.30 \pm 0.26^{* * *}$ \\
\hline \multicolumn{4}{|l|}{ Mean CAL (mm) } \\
\hline Test group & $3.50 \pm 0.45$ & $2.94 \pm 0.50^{* * *}$ & $2.85 \pm 0.42^{* * *}$ \\
\hline Control group & $3.52 \pm 0.44$ & $3.05 \pm 0.44^{* * *}$ & $3.02 \pm 0.45^{* *}$ \\
\hline \multicolumn{4}{|l|}{ BOP (\%) } \\
\hline Test group & $28.8 \pm 31.6$ & $13.5 \pm 7.5^{* *}$ & $11.5 \pm 4.6^{* *}$ \\
\hline Control group & $27.9 \pm 16.5$ & $13.9 \pm 9.6^{* * *}$ & $12.0 \pm 9.2^{* * *}$ \\
\hline \multicolumn{4}{|l|}{ Sites $P D \geq 5$ mm (n) } \\
\hline Test group & $29.88 \pm 15.27$ & $4.16 \pm 7.68^{* * *}$ & $0.64 \pm 1.38^{* * *}$ \\
\hline Control group & $27.62 \pm 6.54$ & $6.54 \pm 8.37^{* * *}$ & $3.12 \pm 4.02 * * * 19$ \\
\hline \multicolumn{4}{|l|}{ API (\%) } \\
\hline Test group & $16.9 \pm 9.7$ & $15.9 \pm 17.7$ & $16.4 \pm 14.4$ \\
\hline Control group & $24.3 \pm 20.7$ & $19.7 \pm 20.7$ & $14.8 \pm 9.6^{*}$ \\
\hline
\end{tabular}

Wilcoxon' signed rank test for paired samples: ${ }^{*} p<0.05,{ }^{* *} p<0.01,{ }^{* * *} p<0.001$, each compared with baseline

Mann Whitney test between the groups: ${ }^{91}<0.05$

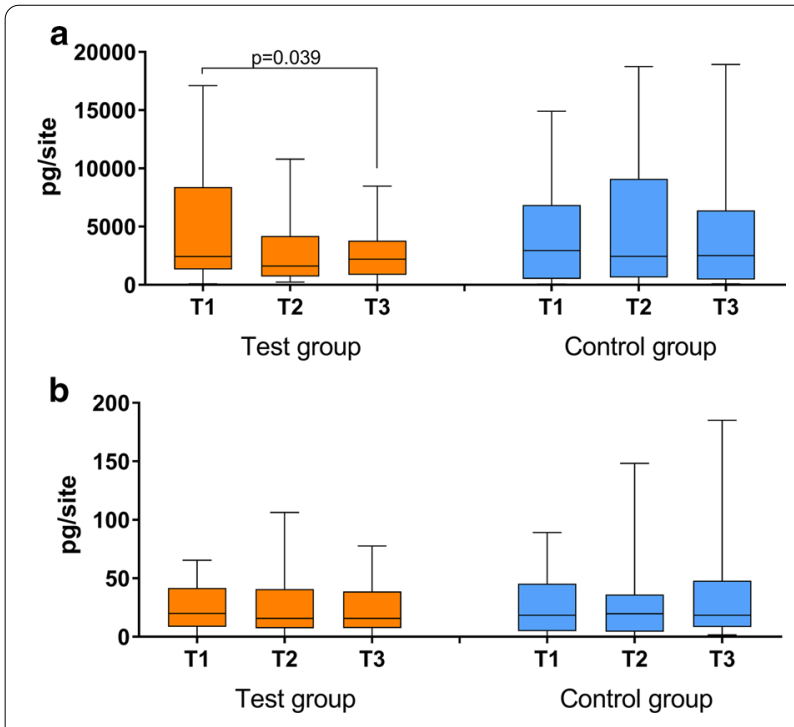

Fig. 3 Levels of MMP-8 (a) and IL-1 $\beta$ (b) in gingival crevicular fluid of the test group (subgingival instrumentation + subgingival erythritol air-polishing) and control group (subgingival instrumentation alone) at baseline (T1) as well as three (T2) and six months (T3) after subgingival instrumentation incl. statistically significant results (Wilcoxon signed rank test for paired samples)
Müller et al. [25] compared an erythritol powder supplemented with chlorhexidine and treatment with ultrasonic debridement during supportive periodontal therapy. They did not find differences in the clinical outcome after 12 months between the groups. Using a similar approach as in our study but with a split-mouth design in 21 patients and a follow-up of three months, there was also no difference between test sites and control sites [14]. In the present study, the statistical unit was the patient. Here differences were also not seen at three months but at six months. It could be assumed that also the supragingival application of air-polishing at three months was beneficial for the outcome at six months.

Glycine powder but not erythritol was applied in other studies. Wennström et al. [26] reported no significant differences of clinical variables between the use of ultrasonic instruments and air polishing with glycine powder and the nozzle device in maintenance patients during the study period of 60 days. Air-polishing with the abrasive glycine powder for $10 \mathrm{~s}$ per periodontal pocket as an adjunctive mean for the SI in the initial therapy of periodontitis did not lead to clinical benefits at plaque and gingival indices, probing depth, bleeding on probing and attachment level 30 days after the treatment when compared with SI only [13]. Tsang et al. [27] found no significant differences for biomarkers after adjunctive airpolishing at SI in untreated periodontitis patients during the study period of six months.

The improvement of the numbers of sites with pathological probing depths $\geq 5 \mathrm{~mm}$ is of clinical importance-shallow pockets without bleeding on probing are considered as pocket closure with stable periodontal conditions [28-30]. Probing depth $\geq 5 \mathrm{~mm}$ is an indicative to schedule the further treatment necessities because sites or teeth with residual $\mathrm{PD} \geq 5 \mathrm{~mm}$ have a higher risk for further attachment loss due to disease progression as well as a higher risk for tooth loss $[28,31]$. The local endpoints of periodontal therapy have been defined with shallow pockets $(\leq 4 \mathrm{~mm})$ without bleeding in patients with $<30 \%$ bleeding sites [32]. In our study we did not find a difference of BOP (primary outcome) between the groups. But the significant lower number of pockets with $\mathrm{PD} \geq 5 \mathrm{~mm}$ six months after the combined treatment in our study suggests a reduced need for repeated SI or surgical periodontal treatment after the combination of SI and air-polishing as described for adjunctive antibiotic treatment during SI [33].

The improvement of PD in the test and control groups was in our study 0.91 and $0.78 \mathrm{~mm}$, respectively. This can be considered as a medium or good improvement. Haffajee et al. [34] had an improvement of $0.45 \mathrm{~mm}$ for PD 

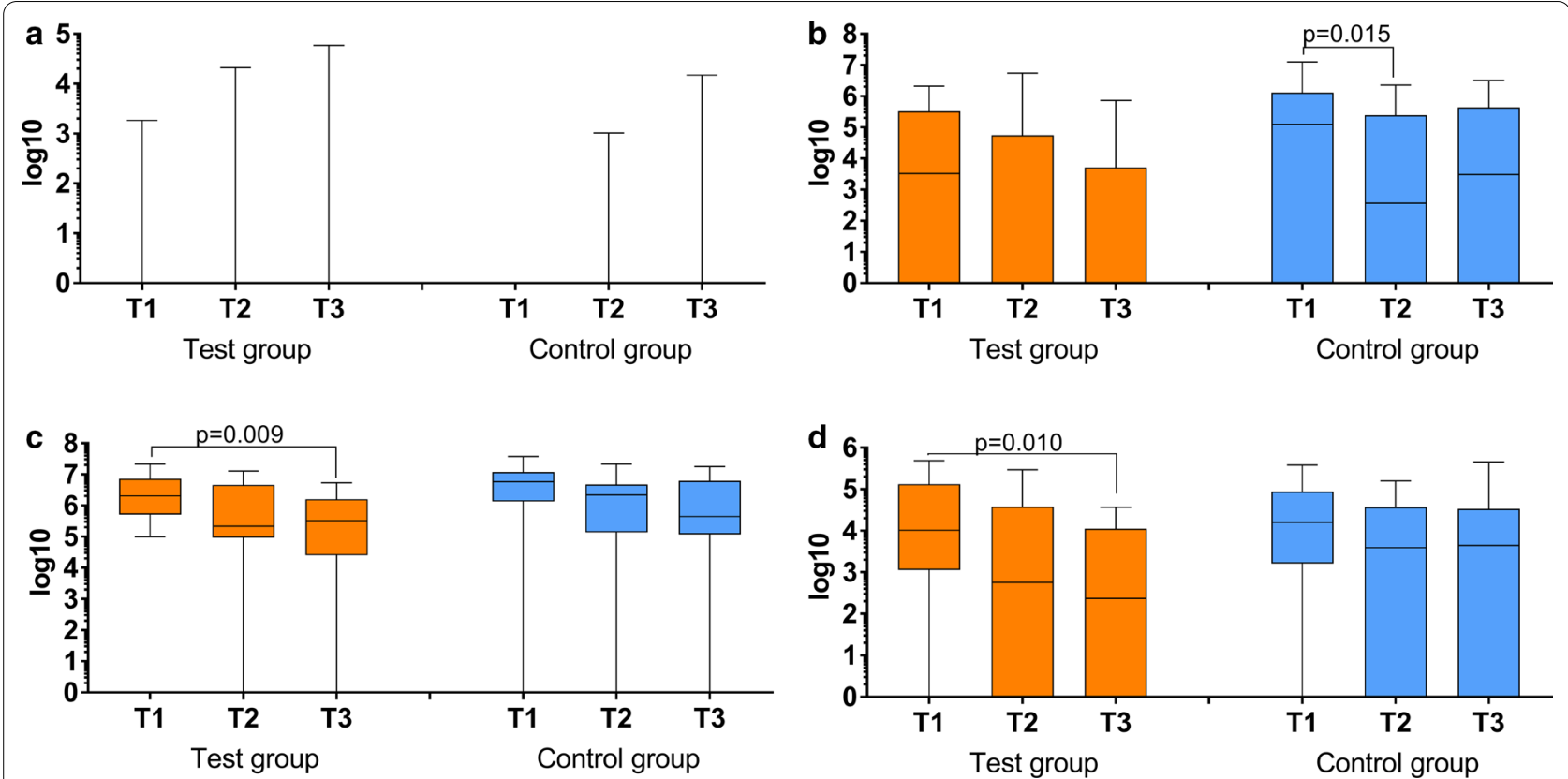

Fig. 4 Counts of Aggregatibacter actinomycetemcomitans (a), Porphyromonas gingivalis (b), Tannerella forsythia (c) and Treponema denticola (d) in the subgingival biofilm of the test group (subgingival instrumentation + subgingival erythritol air-polishing) and control group (subgingival instrumentation alone) at baseline ( $\mathrm{T} 1$ ) as well as three (T2) and six months (T3) after subgingival instrumentation incl. statistically significant results (Wilcoxon signed rank test for paired samples)

after six months and Jentsch et al. [20] improvements of $0.51 / 0.54 \mathrm{~mm}$ after six months.

The improvement of PD in the test group with the adjunctive use of erythritol was $0.13 \mathrm{~mm}$ higher than in the control group. This slightly higher improvement raises the question if it is an indication for the adjunctive use of erythritol applicated by the nozzle system. Taken into account quick and efficient therapy it is obvious that a application time of the nozzle for $5 \mathrm{~s}$ per site as in our study and in other studies [12, 35] instead of $10 \mathrm{~s}$ [13] would be more attractive for the clinician as less time for an effective treatment procedure is needed. On the other hand, the use of the nozzle as a one-way product raises the treatment costs to a certain extent. In addition, it needs some experience to introduce the device properly in the periodontal pocket.

The by $0.13 \mathrm{~mm}$ higher improvement in the test group is in similarity with results by other adjunctive procedures. Differences of $0.10 \mathrm{~mm}$ and $0.19 \mathrm{~mm}$ were reported for the adjunctive application of soft lasers during SI [36, 37]. Luchesi et al. [38] and Malgikar et al. [39] reported a difference of 0.07 and $0.09 \mathrm{~mm}$, respectively, between the improvements of PD after SI with or without adjunctive photodynamic therapy.

At six months, in addition to the clinical variables, the level of MMP-8 was reduced in the test group. Related to air-polishing, MMP-8 level was only once determined in peri-implant sulcus fluid when applying four different cleaning procedures at dental implants after placement [40]; there was no difference to baseline and within the groups at 12 months. At six months, the counts of $T$. denticola and T. forsythia were also reduced only in the test group. Results reported before are different. In patients undergoing supportive therapy there was no difference between groups $[12,35,41]$. Applying air-polishing in periodontal therapy, there were reduced $P$. gingivalis counts when using air-polishing with glycine-powder supragingivally after one month [42]. The only study using airpolishing with erythritol as an adjunct, found decreased P. gingivalis counts at one month, but there was only a follow-up to three months [14]. Full-mouth treatment of systemically and periodontally healthy individuals with air-polishing with glycine powder revealed a significant decrease of bacteria being associated with periodontal disease up to nine days following intervention, however the counts returned to baseline after six weeks [43]. In the present study, the combination of using first subgingivally air-polishing with erythritol and after three months supragingivally may lead to the beneficial results regarding the number of sites with $\mathrm{PD} \geq 5 \mathrm{~mm}$ which seemed to be associated with reduction of T. forsythia and T. denticola. 


\section{Conclusion and limitations}

The study has several limitations like e.g. no occurrence of patients-related factors and no control group with nozzle but without air abrasive powder and not well-balanced distribution of smokers between groups.

Although not showing higher improvements of BOP (primary outcome), the results of our study suggest that subgingival instrumentation with adjunctive erythritol airpolishing powder may add beneficial effects like reducing the number of residual periodontal pocket with $\mathrm{PD} \geq 5 \mathrm{~mm}$ when compared with subgingival instrumentation only and may reduce the need for periodontal surgery.

\section{Abbreviations \\ ${ }^{\circ} \mathrm{C}$ : Degree celsius; AL: Clinical attachment level; API: Interproximal plaque index; BOP: Bleeding on probing; GCF: Gingival crevicular fluid; PD: Probing depth; pg: Picogram; s: Second; SI: Subgingival instrumentation.}

\section{Acknowledgments}

The laboratory work of Anna Magdon and Prashanthnj Sivapatham (Laboratory of Oral Microbiology, University of Bern, Switzerland) is highly acknowledged.

\section{Author's contribution}

All four authors have made substantial contributions to the concept and the design of the study. HJ and SE had the idea of the study. CF and BK collected the data. HJ and SE analysed the data and wrote the manuscript. All authors read and approved the final manuscript.

\section{Funding}

Open Access funding enabled and organized by Projekt DEAL. The study was funded by the participating institutions. The erythritol powder and the AirFlow ${ }^{\circledR}$ Master apparatus with handpieces and nozzles was provided by EMS, Nyon, Switzerland. The funders and the supporting company had no role in study design, data collection and analysis, decision to publish and preparation of the manuscript.

\section{Availability of data and materials}

All data are available from the corresponding author on reasonable request.

\section{Ethics approval and consent to participate}

The study was approved by the Ethics Commission (\#AZ436/16-ek) of the Medical Faculty of the University of Leipzig. All procedures performed in studies involving human participants were in accordance with the ethical standards of the institutional and/or national research committee and with the 1964 Helsinki declaration and its later amendments or comparable ethical standards. Written informed consent was obtained from all individual participants included in the study. The manuscript adheres to CONSORT guidelines.

\section{Consent for publication}

Not applicable.

\section{Competing interests}

The authors (Holger Jentsch, Christian Flechsig, Benjamin Kette and Sigrun Eick) declare that they have no competing interests. Sigrun Eick is associate editor of BMC Oral health.

\section{Author details}

1 Department of Cariology, Endodontology and Periodontology, Centre for Periodontology, University Hospital of Leipzig, Liebigstr. 12, Haus 1, 04103 Leipzig, Germany. ${ }^{2}$ Private Dental Practice, Berlin, Germany. ${ }^{3}$ Department of Periodontology, School of Dental Medicine, University of Bern, Bern, Switzerland.
}

Received: 27 July 2020 Accepted: 10 December 2020

Published online: 29 December 2020

\section{References}

1. Bartold PM, Van Dyke TE. Periodontitis: a host-mediated disruption of microbial homeostasis. Unlearning learned concepts. Periodontology. 2000;2013(62):203-17.

2. Grossi SG, Zambon JJ, Ho AW, Koch G, Dunford RG, Machtei EE, Norderyd OM, Genco RJ. Assessment of risk for periodontal disease. I. Risk indicators for attachment loss. J Periodontol. 1994;65:260-7.

3. Lang NP, Bartold PM. Periodontal health. J Periodontol. 2018;89(Suppl 1):S9-16.

4. Darveau RP, Hajishengallis G, Curtis MA. Porphyromonas gingivalis as a potential community activist for disease. J Dent Res. 2012;91:816-20.

5. Sanz I, Alonso B, Carasol M, Herrera D, Sanz M. Nonsurgical treatment of periodontitis. J Evid Based Dent Pract. 2012;12(3 Suppl):76-86.

6. Cobb CM. Clinical significance of non-surgical periodontal therapy: an evidence-based perspective of scaling and root planing. J Clin Periodontol. 2002;29(Suppl 2):6-16.

7. Suvan J, Leira Y, Moreno F, Graziani F, Derks J, Tomasi C. Subgingival instrumentation for treatment of periodontitis. A systematic review. J Clin Periodontol. 2020;47:155-75. https://doi.org/10.1111/JCPE.13245 (Epub ahead of print).

8. Cobb CM, Daubert DM, Davis K, Deming J, Flemmig TF, Pattison A, Roulet JF, Stambaugh RV. Consensus conference findings on supragingival and subgingival air polishing. Compend Contin Educ Dent. 2017;38:e1-4.

9. Munro IC, Berndt WO, Borzelleca JF, Flamm G, Lynch BS, Kennepohl E, Bär EA, Modderman J. Erythritol: an interpretive summary of biochemical, metabolic, toxicological and clinical data. Food Chem Toxicol. 1998;36:1139-74.

10. Hägi TT, Hofmänner P, Salvi GE, Ramseier CA, Sculean A. Clinical outcomes following subgingival application of a novel erythritol powder by means of air polishing in supportive periodontal therapy: a randomized, controlled clinical study. Quintessence Int. 2013;44:753-61.

11. Hashino E, Kuboniwa M, Alghamdi SA, Yamaguchi M, Yamamoto R, Cho $\mathrm{H}$, Amano A. Erythritol alters microstructure and metabolomic profiles of biofilm composed of Streptococcus gordonii and Porphyromonas gingivalis. Mol Oral Microbiol. 2013;28:435-51.

12. Hägi TT, Hofmänner P, Eick S, Donnet M, Salvi GE, Sculean A, Ramseier CA. The effects of erythritol air-polishing powder on microbiologic and clinical outcomes during supportive periodontal therapy: six-month results of a randomized controlled clinical trial. Quintessence Int. 2015;46:31-41.

13. Caygur A, Albaba MR, Berberoglu A, Yilmaz HG. Efficacy of glycine powder air-polishing combined with scaling and root planing in the treatment of periodontitis and halitosis: a randomised clinical study. J Int Med Res. 2017;45:1168-74.

14. Park EJ, Kwon EY, Kim HJ, Lee JY, Choi J, Joo JY. Clinical and microbiological effects of the supplementary use of an erythritol powder air-polishing device in non-surgical periodontal therapy: a randomized clinical trial. J Periodontal Implant Sci. 2018;48:295-304.

15. Armitage GC. Development of a classification system for periodontal diseases and conditions. Ann Periodontol. 1999;4:1-6.

16. Tonetti MS, Greenwell H, Kornman KS. Staging and grading of periodontitis: framework and proposal of a new classification and case definition. J Periodontol. 2018;89(Suppl 1):S159-72.

17. Lange DE, Plagmann HC, Eenboom A, Promesberger A. Clinical methods for the objective evaluation of oral hygiene (in German). Dtsch Zahnarztl Z. 1977;32:44-7.

18. Chondros P, Nikolidakis D, Christodoulides N, Rössler R, Gutknecht N, Sculean A. Photodynamic therapy as adjunct to non-surgical periodontal treatment in patients on periodontal maintenance: a randomized controlled clinical trial. Lasers Med Sci. 2009;24:681-8.

19. Jentsch H, Buchmann A, Friedrich A, Eick S. Nonsurgical therapy of chronic periodontitis with adjunctive systemic azithromycin or amoxicillin/metronidazole. Clin Oral Investig. 2016;20:1765-73.

20. Jentsch HFR, Heusinger T, Weickert A, Eick S. Professional tooth cleaning prior non-surgical periodontal therapy: a randomized clinical trial. J Periodontol. 2020;91:174-82. 
21. Sanz M, Herrera D, Kebschull M, Chapple I, Jepsen S, Beglundh T, Sculean A, Tonetti MS. Treatment of stage I-III periodontitis-The EFP S3 level clinical practice guideline. J Clin Periodontol. 2020;47(Suppl 22):4-60.

22. Griffiths GS. Formation, collection and significance of gingival crevice fluid. Periodontology. 2000;2003(31):32-42.

23. Moher D, Schulz KF, Altman DG. The CONSORT statement: revised recommendations for improving the quality of reports of parallel-group randomised trials. Lancet. 2001;357:1191-4.

24. Kruse AB, Maamar R, Akakpo DL, Woelber JP, Wittmer A, Vach K, RatkaKrüger P, Al-Ahmad A. Effects of subgingival air-polishing with trehalose powder on oral biofilm during periodontal maintenance therapy: a randomized-controlled pilot study. BMC Oral Health. 2020;20:123.

25. Müller N, Moëne R, Cancela JA, Mombelli A. Subgingival air-polishing with erythritol during periodontal maintenance: randomized clinical trial of twelve months. J Clin Periodontol. 2014;41:883-9.

26. Wennström JL, Dahlén G, Ramberg P. Subgingival debridement of periodontal pockets by air polishing in comparison with ultrasonic instrumentation during maintenance therapy. J Clin Periodontol. 2011;38:820-7.

27. Tsang YC, Corbet EF, Jin $\sqcup$. Subgingival glycine powder air-polishing as an additional approach to nonsurgical periodontal therapy in subjects with untreated chronic periodontitis. J Periodontal Res. 2018;53:440-5.

28. Chapple ILC, Mealey BL, Van Dyke TE, Bartold PM, Dommisch H, Eickholz P, Geisinger ML, Genco RJ, Glogauer M, Goldstein M, Griffin TJ, Holmstrup P, Johnson GK, Kapila Y, Lang NP, Meyle J, Murakami S, Plemons J, Romito GA, Shapira L, Tatakis DN, Teughels W, Trombelli L, Walter C, Wimmer $\mathrm{G}$, Xenoudi P, Yoshie H. Periodontal health and gingival diseases and conditions on an intact and a reduced periodontium: consensus report of workgroup 1 of the 2017 World Workshop on the Classification of Periodontal and Peri-Implant Diseases and Conditions. J Clin Periodontol. 2018;45(Suppl 20):S68-77.

29. Figuero E, Roldan S, Serrano J, Escribano M, Martin C, Preshaw PM. Efficacy of adjunctive therapies in patients with gingival inflammation. A systematic review and meta-analysis. J Clin Periodontol. 2020;47:125-43. https://doi.org/10.1111/jcpe.13244 (Epub ahead of print).

30. Tomasi C, Leyland AH, Wennström JL. Factors influencing the outcome of non-surgical periodontal treatment: a multilevel approach. J Clin Periodontol. 2007;34:682-90.

31. Matuliene G, Pjetursson BE, Salvi GE, Schmidlin K, Brägger U, Zwahlen M, Lang NP. Influence of residual pockets on progression of periodontitis and tooth loss: results after 11 years of maintenance. J Clin Periodontol. 2008;35:685-95.

32. Loos BG, Needleman I. Endpoints of active periodontal therapy. J Clin Periodontol. 2020. https://doi.org/10.1111/jcpe.13253 (Epub ahead of print).

33. Cionca N, Giannopoulou C, Ugolotti G, Mombelli A. Amoxicillin and metronidazole as an adjunct to full-mouth scaling and root planing of chronic periodontitis. J Periodontol. 2009;80:364-71.
34. Haffajee AD, Cugini MA, Dibart S, Smith C, Kent RL Jr, Socransky SS. The effect of SRP on the clinical and microbiological parameters of periodontal diseases. J Clin Periodontol. 1997:24:324-34.

35. Kargas K, Tsalikis L, Sakellari D, Menexes G, Konstantinidis A. (1997) Pilot study on the clinical and microbiological effect of subgingival glycine powder air polishing using a cannula-like jet. Int J Dent Hyg. 1997;13:161-9.

36. Aykol G, Baser U, Maden I, Kazak Z, Onan U, Tanrikulu-Kucuk S, Ademoglu $\mathrm{E}$, Issever $\mathrm{H}$, Yalcin $\mathrm{F}$. The effect of low-level laser therapy as an adjunct to non-surgical periodontal treatment. J Periodontol. 2011;82:481-8.

37. Lopes BM, Theodoro LH, Melo RF, Thompson GM, Marcantonio RA. Clinical and microbiologic follow-up evaluations after non-surgical periodontal treatment with erbium:YAG laser and scaling and root planing. J Periodontol. 2010:81:682-91.

38. Luchesi VH, Pimentel SP, Kolbe MF, Ribeiro FV, Casarin RC, Nociti FH Jr, Sallum EA, Casati MZ. Photodynamic therapy in the treatment of class II furcation: a randomized controlled clinical trial. J Clin Periodontol. 2013:40:781-8.

39. Malgikar S, Reddy SH, Sagar SV, Satyanarayana D, Reddy GV, Josephin JJ. Clinical effects of photodynamic and low-level laser therapies as an adjunct to scaling and root planing of chronic periodontitis: a split-mouth randomized controlled clinical trial. Indian J Dent Res. 2016;27:121-6

40. Schmidt KE, Auschill TM, Sculean A, Arweiler NB. Clinical evaluation of non-surgical cleaning modalities on titanium dental implants during maintenance care: a 1-year follow-up on prosthodontic superstructures. Clin Oral Investig. 2019:23:1921-30.

41. Moëne R, Décaillet F, Andersen E, Mombelli A. Subgingival plaque removal using a new air-polishing device. J Periodontol. 2010;81:79-88.

42. Flemmig TF, Arushanov D, Daubert D, Rothen M, Mueller G, Leroux BG. Randomized controlled trial assessing efficacy and safety of glycine powder air polishing in moderate-to-deep periodontal pockets. J Periodontol. 2012;83:444-52.

43. Reinhardt B, Klocke A, Neering SH, Selbach S, Peters U, Flemmig TF, Beikler T. Microbiological dynamics of red complex bacteria following full-mouth air polishing in periodontally healthy subjects-a randomized clinical pilot study. Clin Oral Investig. 2019;23:3905-14.

\section{Publisher's Note}

Springer Nature remains neutral with regard to jurisdictional claims in published maps and institutional affiliations.
Ready to submit your research? Choose BMC and benefit from:

- fast, convenient online submission

- thorough peer review by experienced researchers in your field

- rapid publication on acceptance

- support for research data, including large and complex data types

- gold Open Access which fosters wider collaboration and increased citations

- maximum visibility for your research: over 100M website views per year

At BMC, research is always in progress.

Learn more biomedcentral.com/submissions 